Revue européenne des migrations internationales

vol. 35 - $n^{\circ} 3$ et $4 \mid 2019$

Danses, musiques et (trans)nationalismes

\title{
Feldman Nehara, Migrantes: du bassin du fleuve \\ Sénégal aux rives de la Seine
}

Suzanne Dioly-Niang et Claire Lévy-Vroelant

\section{(2) OpenEdition}

Édition électronique

URL : https://journals.openedition.org/remi/13971

DOI : 10.4000/remi. 13971

ISSN : $1777-5418$

Éditeur

Université de Poitiers

\section{Édition imprimée}

Date de publication : 1 décembre 2019

Pagination : 299-301

ISBN : 979-10-90426-65-8

ISSN : 0765-0752

\section{Référence électronique}

Suzanne Dioly-Niang et Claire Lévy-Vroelant, «Feldman Nehara, Migrantes : du bassin du fleuve Sénégal aux rives de la Seine ", Revue européenne des migrations internationales [En ligne], vol. 35 - n³ et 4 |

2019, mis en ligne le 01 décembre 2019, consulté le 15 avril 2022. URL : http://

journals.openedition.org/remi/13971 ; DOI : https://doi.org/10.4000/remi.13971

Ce document a été généré automatiquement le 15 avril 2022.

(c) Université de Poitiers 


\title{
Feldman Nehara, Migrantes: du bassin du fleuve Sénégal aux rives de la Seine
}

\author{
Suzanne Dioly-Niang et Claire Lévy-Vroelant
}

\section{RÉFÉRENCE}

Feldman Nehara (2018) Migrantes: du bassin du fleuve Sénégal aux rives de la Seine, Paris, La Dispute, 224 p., ISBN : 978-2-84303-292-9

1 Le livre de Nehara Feldman pose d'emblée une question qui, sans être nouvelle, éclaire de façon particulièrement intéressante la position sociale des femmes migrantes : que fait la migration aux rapports de genre ? L'enquête, et c'est sa force, s'articule sur trois lieux, au village, à Bamako et en région parisienne. Les migrantes rencontrées sont toutes d'origine rurale et ont la particularité d'appartenir au lignage Dagnoko. L'auteure a vécu plusieurs mois dans le lou-o (unité domestique) de Kalilou Dagnoko, représentant du dit lignage, fondateur du village de Galoba et à ce titre considéré comme «noble » et strictement endogame. À partir de Galoba où il tient une position dominante, ce lignage est dispersé, avec des parents à Bamako, mais aussi dans d'autres capitales africaines ainsi qu'en Europe, particulièrement en Espagne et en France. Le choix des trois lieux permet de saisir comment les négociations des positions sociales inscrivent les rapports de genre dans des processus complexes «de dominations, de résistances et d'accommodements toujours révisés » (p. 202). Agrémentée de dessins que l'on souhaiterait plus nombreux, l'enquête n'évite pas les heurs et malheurs des relations entre les femmes elles-mêmes. On trouve dans le livre des passages particulièrement éclairants sur l'exploitation dont sont victimes les jeunes filles de la part de leurs ainées, la règle virilocale renforçant leur dépendance : éternelle migrante, la fille est destinée à quitter le lou-o de son père pour rejoindre celui d'une sœur ainée, d'une tante ou d'un mari. Comment s'attacherait-elle à un lieu? On apprend que les femmes voyagent, avant et après la migration. Une fois émigrées, elles se déplacent 
aussi "pour affaires ", et parfois très loin. Ce qui ne manque pas de questionner sur l'apparente contradiction entre l'extrême contrôle dont elles font l'objet dans le village, et ces intermèdes de liberté.

Le livre comprend trois parties : la première interroge le lien entre genre et espace dans les lieux du village, au sein du lou-o habité et au dehors. La division sexuelle de l'espace est analysée finement et montre une division genrée parfaitement inégalitaire : car si les lieux du travail (cuisine, points d'eau, espace intérieur de plain-pied et topographiquement inférieurs du lou-o) sont ceux des femmes, les hommes quant à eux sont chez eux partout, à la fois dehors, au village, mais aussi dans la cour et dans les espaces des femmes le cas échéant. Seule la chambre est privée, mais le plus souvent partagée avec d'autres femmes de la même condition, c'est-à-dire à la même place dans la hiérarchie complexe résultant de l'âge et de la filiation. Leurs investissements dans l'ameublement et la décoration deviennent signes et enjeux de respectabilité et indices patents de la réussite du mari. Mais les épouses n'en restent pas moins sous la menace d'une expulsion toujours possible, ou de l'arrivée d'une coépouse.

Une telle division se reproduit-elle en ville, à Bamako, en région parisienne? La deuxième partie qui croise genre et mobilité fait apparaitre l'ambigüité qui caractérise la position des filles et des femmes migrantes. Elle balaye au passage l'idée reçue selon laquelle les filles et les femmes se déplaceraient pour des raisons familiales, notamment pour se marier, et met en lumière une redistribution liée au travail domestique que les filles et les épouses accomplissent dans les demeures successives où les pères et les maris les affectent. La troisième partie explore le paradoxe de deux positionnements concurrents, mais qu'il leur faut faire maintenir ensemble, entre un «ici » en bas de l'échelle sociale et un «là-bas» où les responsabilités parfois écrasantes s'accompagnent d'un statut privilégié.

4 Les apports du livre sont multiples, nous en soulignerons les principaux. D'abord, l'analyse de la division de l'espace au village est remarquable, ne cédant ni aux idées reçues ni au romantisme d'une vision d'espaces exclusivement féminins. Ensuite, l'analyse des rapports de genre, avant et pendant le processus migratoire, articule de façon convaincante les relations de pouvoir et de domination entre femmes avec ceux qui règlent les rapports entre groupes d'âge (des ainées qui exploitent leurs cadettes) entre classes (nobles, personnes libres, castes et esclaves), entre milieux géographiques (village, Bamako, France).

5 Comme dans toute recherche bien construite, l'auteure évoque ses difficultés en tant que chercheure dans un contexte africain où «parler de ses réussites est perçu comme de la vantardise ", mais où évoquer ses échecs n'est pas plus accepté et où dévoiler ses projets risque de vous porter malheur. Tenter de recueillir des traces de vie et des récits d'expériences peut s'avérer maladroit ou suspect : la jeune femme blanche venue de France fait figure de représentante de la bureaucratie « dans un climat de chasse aux sans-papiers ». Mais de cette position, Nehera Feldman a su tirer avantage. Elle découvre - point capital - que sa position d'Occidentale, d'intellectuelle et de blanche la met hors du groupe des femmes. Une place «à part » lui est réservée dans le lou-o : elle a dû batailler pour se tenir avec les femmes alors qu'on la traitait comme une invitée, presque un homme. Cette particularité l'amène à mieux appréhender la position des migrantes de retour au village : fortes de leur pouvoir financier - le livre s'ouvre sur une scène édifiante, où la mariée immigrée « sort de son sac un gros paquet de billets de vingt euros qu'elle commence à distribuer autour d'elle comme des 
morceaux de papier sans valeur » (p.9) - les migrantes jouissent de privilèges qui en font des quasi-hommes, les dispensent des tâches domestiques et les placent au-dessus des normes habituelles de commensalité. Ce placement «à part» des migrantes réduirait les chances que leur conduite ne débouche sur une remise en question plus globale de la domination des hommes sur les femmes, et des femmes de rang ou d'âge supérieur sur les autres femmes. En autorisant la transgression, on sauve l'ordre établi.

Pour ces personnes, femmes plutôt, mais aussi hommes, rencontrés par Nehara Feldman, la migration permet d'acquérir une position privilégiée dans le pays de départ grâce aux moyens financiers qu'elle procure. Mais l'auteure est allée enquêter de l'autre côté de la migration. Ses constats non seulement démentent, comme on le sait depuis les travaux de Sayad, le bonheur de l'immigré, mais ils invalident partiellement le credo de l'émancipation des femmes par la migration. Toujours prisonnières des lois de la lignée, les femmes brillent au pays tout en souffrant en France des mêmes soumissions que leurs sœurs au village, voire à Bamako. L'autorité des pères et des maris ne semble pas faiblir sous la distance et la peur de déchoir en désobéissant amène les épouses à accepter y compris la violence physique à leur encontre. Alors qu'épouser un migrant a pu constituer une échappatoire et éviter d'être réduite à une maind'œuvre gratuite au service des belles-mères, l'espoir né de l'image que renvoient les migrante-s durant leurs visites se révèle illusoire sans que pour autant les migrantes y renoncent quand elles sont elles-mêmes en position de le conforter.

$7 \quad$ Si au village les femmes réduisent leurs sorties au strict cadre des motifs légitimés et des événements familiaux sous peine de ruiner leur réputation, en France, la solitude et la crainte de l'inconnu limitent encore davantage leur présence dans l'espace public. L'espace intérieur où la présence des femmes est légitime se retrouve réduit : la grande maison est remplacée par un appartement, parfois même par un studio ou une chambre d'hôtel. Tandis que les hommes passent leur temps à l'extérieur, les femmes sont assignées à résidence de façon d'autant plus radicale que leur séjour est irrégulier. Aussi, contrairement à la thèse de l'émancipation par le collectif, les observations de l'auteure donnent plutôt à voir l'isolement et l'absence de solidarité entre les femmes. Les gains accumulés par des activités commerciales sont jalousement conservés hors de portée du mari et serviront à assurer la survie et l'indépendance de la femme en cas de rupture de l'union, mais aussi à satisfaire les sollicitations incessantes de la famille, des voisins et des amies, afin de maintenir le prestige lié à leur condition.

Une rupture de taille se manifeste cependant en France avec l'accès des femmes à l'emploi salarié qui bouscule la représentation du travail domestique. Mais contrairement au Mali, le nombre de femmes dans le foyer est réduit et le recours à une domestique impossible. Aussi, la réorganisation des tâches ménagères au sein du couple s'impose, tout comme dans le recours à la contraception avec ou sans l'accord du conjoint. Alors que les normes du milieu rural continuent à être valorisées, les femmes migrantes s'en éloignent progressivement dans la vie réelle. Finalement, malgré la sécheresse des conclusions de chapitre et un suivi dans le temps en pointillé de ses informatrices, Nehara Feldman nous livre une enquête instructive, grâce à laquelle on comprend que la mise en scène festive et ostentatoire de leur réussite participe de la perpétuation de l'ordre existant au pays et les dédommage des souffrances de l'immigrée, pour reprendre l'expression de Sayad. 


\section{AUTEURS}

\section{SUZANNE DIOLY-NIANG}

Sociologue, Doctorante, Université Paris 8

\section{CLAIRE LEVY-VROELANT}

Sociologue, Professeure, Université Paris 8 